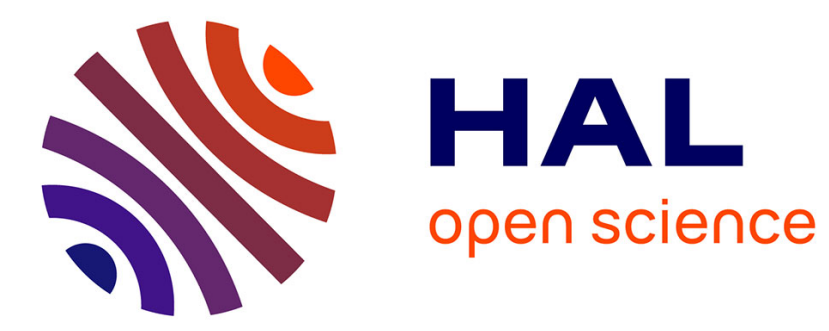

\title{
Computational Investigation of the Effective Mechanical Behavior for 3D Pre-Buckled Auxetic Lattices
}

Frederic Albertini, Justin Dirrenberger, Andrey Molotnikov, Cyrille Sollogoub

\section{To cite this version:}

Frederic Albertini, Justin Dirrenberger, Andrey Molotnikov, Cyrille Sollogoub. Computational Investigation of the Effective Mechanical Behavior for 3D Pre-Buckled Auxetic Lattices. Journal of Applied Mechanics, 2019, 86 (11), pp.111003. 10.1115/1.4044542 . hal-02370867

\section{HAL Id: hal-02370867 https://hal.science/hal-02370867}

Submitted on 19 Nov 2019

HAL is a multi-disciplinary open access archive for the deposit and dissemination of scientific research documents, whether they are published or not. The documents may come from teaching and research institutions in France or abroad, or from public or private research centers.
L'archive ouverte pluridisciplinaire HAL, est destinée au dépôt et à la diffusion de documents scientifiques de niveau recherche, publiés ou non, émanant des établissements d'enseignement et de recherche français ou étrangers, des laboratoires publics ou privés. 
Frederic Albertini

Laboratoire PIMM,

Arts \& Métiers-ParisTech, Cnam, CNRS,

75013 Paris, France

e-mail: frederic.albertini@ensam.eu

Justin Dirrenberger ${ }^{1}$

Associate Professor

Laboratoire PIMM,

Arts \& Métiers-ParisTech, Cnam, CNRS,

75013 Paris, France

e-mail: justin.dirrenberger@ensam.eu

Andrey Molotnikov

Department of Materials Science and Engineering, Monash University,

Clayton, VIC 3800, Australia

e-mail: andrey.molotnikov@monash.edu

\section{Cyrille Sollogoub}

Professor

Laboratoire PIMM,

Arts \& Métiers-ParisTech, Cnam, CNRS,

75013 Paris, France

e-mail: cyrille.sollogoub@ensam.eu

\section{Computational Investigation of the Effective Mechanical Behavior for 3D Pre-Buckled Auxetic Lattices}

\begin{abstract}
Negative Poisson's ratio materials, or auxetics, have drawn attention for the past 30 years. The auxetic effect could lead to improved mechanical properties such as acoustic damping, indentation resistance, or crashworthiness. In this work, two 3D auxetic lattices are introduced. Auxeticity is achieved by design through pre-buckling of the lattice struts. The influence of geometrical parameters on the effective elastic properties is investigated using computational homogenization method with periodic boundary conditions. Effective Young's modulus is 3D mapped to reveal anisotropy and identify spatial orientations of interest. The effective Poisson ratio is computed for various geometric configurations to characterize auxeticity. Finally, the influence of effective elastic properties on energy dissipation under compression is explored for elastoplastic lattices with different loading directions, using finite element simulations. Results suggest that loading $3 D$ auxetic lattices along their stiffest direction maximizes their crashworthiness. [DOI: 10.1115/1.4044542]
\end{abstract}

Keywords: lattice, auxetic, computational homogenization, architectured materials, crashworthiness, computational mechanics, elasticity, mechanical properties of materials, plasticity

\section{Introduction}

Architectured materials are a rising class of advanced materials that bring new possibilities in terms of functional properties, filling the gaps and pushing the limits of Ashby's materials performance maps [1]. The term architectured materials encompasses any material obtained through a design process aiming at fulfilling a specific set of requirements, in terms of functionality, behavior, or performance, induced by a particular morphology, i.e., the relative topological arrangement between multiple phases, such that some of its materials properties, e.g., yield strength/density, are improved in comparison with those of its constituents due to structure and composite effects [1-5].

Auxetics are a subclass of architectured materials exhibiting a negative Poisson's ratio, i.e., they become larger when stretched and thinner when compressed, contrary to conventional materials with a positive Poisson's ratio. Material stability requires the tensor of elastic moduli to be positive definite, resulting in positive Young's modulus, $E$, and Poisson's ratio $\nu$ ranging from -1 , for unshearable materials, to 0.5 , for incompressible or rubber-like materials, in the case of three-dimensional isotropic elasticity. Although most materials naturally exhibit a positive Poisson's ratio, auxetics [6] have been engineered since the 1980s [7-9]. These materials have been drawing attention since then [10-27], and a number of potential applications were proposed [28-33]. Auxetic materials can also enhance acoustic damping [34], which was shown experimentally in Refs. [35-38]. The use of auxetics as building blocks for wave-guiding metamaterials has also been investigated in Refs. [39-42]. Moreover, experiments on deterministic and stochastic auxetic foams seem to provide evidence of improved crashworthiness [43-46], as well as better indentation resistance $[22,47]$ in comparison with conventional cellular materials.

To exploit the possibilities offered by auxetics, effective mechanical behavior models are needed for such materials to be used in

\footnotetext{
${ }^{1}$ Corresponding author
}

industrial structural applications, i.e., to be considered from an equivalent continuum viewpoint in structural calculations. To do so, analytical strategies are usually favored due to their efficiency and easy implementation. Nevertheless, they rely on strong hypotheses that can be limiting in terms of applicability to complex cell topologies, as well as nonlinear constitutive behavior. To study the elastoplastic response of metallic auxetics as it has been done numerically [21,48] and experimentally [49], it appears that fullfield finite element analysis combined with computational homogenization is a powerful approach [50-56].

The present work introduces two novel three-dimensional periodic auxetic structures. Auxetic behavior is achieved by design, considering unit cells with pre-buckled struts. Computational homogenization using periodic boundary conditions is considered here in the case of periodic architectured materials to determine the effective elastic properties of the two proposed auxetic cells with varying geometrical parameters.

First, a parametric geometrical description of the unit cells is given. After introducing the computational strategy, the influence of parameters on the effective elastic properties is examined based on computational homogenization. An extension to elastoplasticity is explored to evaluate the interest of the pre-buckled auxetic designs on plastic work and crashworthiness. Results are then discussed and put into perspective with the available literature on auxetics. Finally, conclusions are drawn, and perspectives on further extensions of the present work are made.

Throughout this work, the following notation is used: $x$ for scalars, $x$ for vectors, $\boldsymbol{x}$ for second-order tensors, $\boldsymbol{x}$ for fourthorder tensors, $\cdot$ for dot product, and : for doubly côntracted dot product.

\section{Auxetic Lattices}

Among architectured materials, lattices are a combination of material and space. They are structures composed of a connected network of struts, which may be organized periodically in space. They are generally used in cases where there is a need for high specific stiffness or high specific strength [57-69]. 
In 1864, J. C. Maxwell gave the first general method for the static analysis of truss frameworks [70], based on the thermodynamical considerations of energy conservation, and Clapeyron's theorem, i.e., the elastic energy of a strut being equal to the sum of the mechanical works of external forces. Following Maxwell and the generalization of his criterion for self-stressed truss frameworks by Refs. [58,71] showed that depending on the network connectivity, lattice structures can be broken down into two separate groups: stretch-dominated and bending-dominated structures. Such that, for 3D lattices, it yields Eq. (1):

$$
M=b-3 j+6=s-m
$$

where $b$ is the number of struts in a cell, $j$ is the number of frictionless vertices, $s$ is the number of self-stressed members, and $m$ is the number of mechanisms, both of the latter can be determined by finding the rank of the equilibrium matrix describing the framework in a full structural analysis [72]. If $M<0$, the structure is bending dominated; if $M \geq 0$, the structure is stretch dominated.

Auxetic lattices are known to be bending dominated to enable re-entrant or rolling-in mechanisms [73,74]. Therefore, mechanical analysis of truss frameworks can be useful for guiding the design process of auxetics. Pertinent choice of morphology and/or topology design can be made to attain specific mechanical properties [75-77]. The design of these two novel unit cells was inspired by the works of Dirrenberger et al. [22] and Warmuth et al. [78]. In Ref. [78], the authors create an auxetic unit cell based on the analysis of eigenmodes for a cubic unit cell. The unit cell created was, therefore, buckled by design, to grant auxetic properties. Buckling-induced auxeticity, i.e., taking advantage of structural instabilities, has also been explored in the literature [25,79-82]. In this work, we are considering two periodic pre-buckled unit cells that stem from the face-centered cubic (FCC) unit cell, as shown in Fig. 1.

By curving all struts of the FCC cell inward (see equations in Appendix A), it is expected that the resulting design will exhibit negative Poisson's ratio, for the hexatruss cell developed in Ref. [22]: by enforcing buckling orientations of the constituting struts, it is possible to select and tune the deformation behavior of unit cells. Since they are already buckled, it is also expected that those cells will be softer than a regular FCC cell, with an influence of the pre-buckling amplitude.

These assumptions will be assessed using the computational homogenization method to identify elastic moduli tensors for different geometric configurations of the cells.

2.1 Parametric Description. In this section, both unit cells and limiting values are described with regards to their geometrical parameters.

2.1.1 Hexaround Unit Cell. This cell is composed of cylindric struts that are curvilinear, arc-shaped. The arcs are oriented inward, thus creating a re-entrant effect when the structure is being compressed. Conversely, the struts are expected to straighten when stretched.
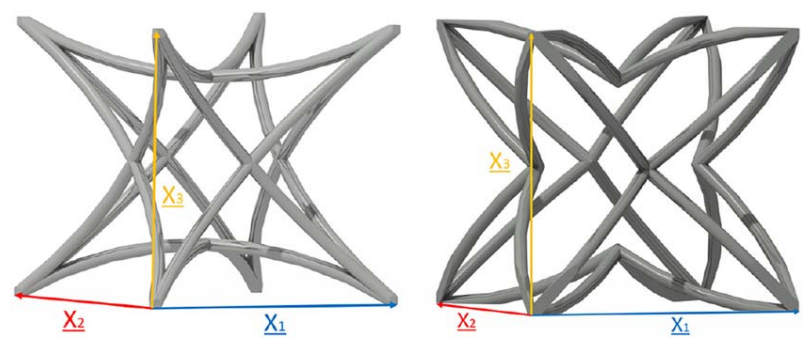

Fig. 1 Hexaround (left) and inverse hexaround (right) unit cells, with their periodicity vectors $\underline{X}_{i}$
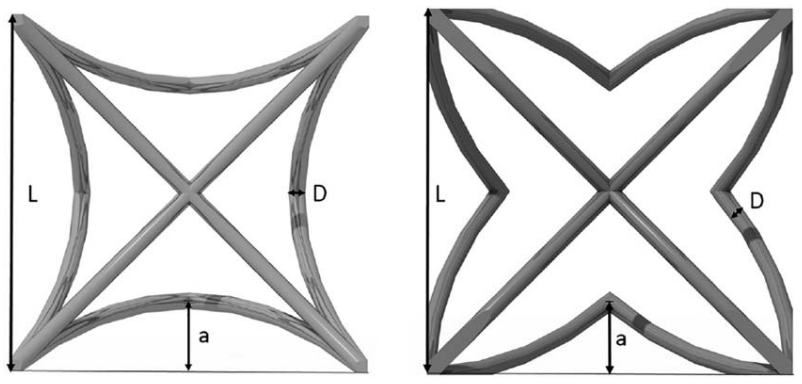

Fig. 2 Hexaround (left) and inverse hexaround (right) geometric descriptions

The hexaround unit cell can be described using three parameters: $L$, side length of the cubic cell, $a$, distance between top and bottom of arcs, and $D$, diameter of the struts (see Fig. 2). These parameters are limited by the following geometrical boundaries:

$$
\begin{aligned}
& L \in] 0 ;+\infty[ \\
& a \in] 0 ; L / 4] \\
& D \in] 0 ; 2 a]
\end{aligned}
$$

Parameter $a$ represents the buckling amplitude: for $a=0$, the cell correspond to a regular FCC cell. For $a=L / 4$, struts are merged in the corners of the cell. From $a>L / 4$, intersection of the struts occurs, thus generating a new geometry (see Fig. 3) with different mechanical behaviors, out of the scope of the present study. Even though every combinations inside these parameter ranges are geometrically possible, some of them also lead to geometrical configurations presenting nonauxetic behavior.

2.1.2 Inverse Hexaround Unit Cell. The inverse hexaround unit cell has been obtained starting from the hexaround by reversing the curvature; struts then intersect at the center of sides, at the buckling amplitude $a$. The inverse hexaround unit cell is described using the same three parameters: $L, a$, and $D$ (see Fig. 2). Again, the parameters can vary within the following ranges:

$$
\begin{aligned}
& L \in] 0 ;+\infty[ \\
& a \in] 0 ; L / 2[ \\
& D \in] 0 ; 2 a]
\end{aligned}
$$

In the same way as the hexaround cell, when $a=0$, this cell corresponds to a regular FCC cell. From $a>L / 2$, the topology and nodal connectivity change due to strut intersection. Every combination within the parameter ranges is geometrically possible, but some of them also lead to geometries exhibiting nonauxeticity.
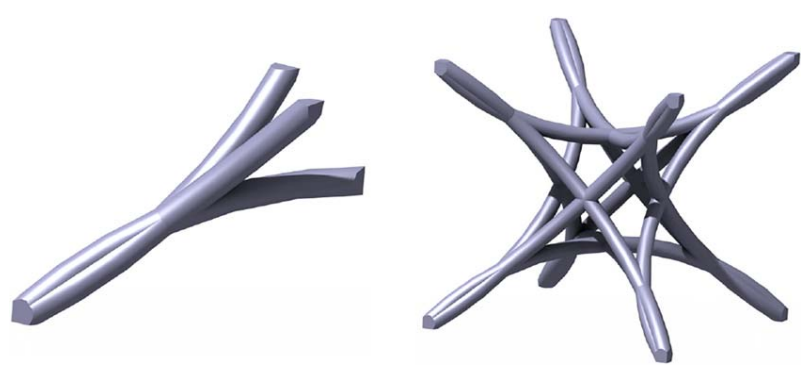

Fig. $3 \quad 1 / 8$ of cell with intersection (left) and resulting cell (right) 
Having introduced the two unit cell geometries, and range of varying parameters, it is noteworthy that effective elastic properties of periodic auxetic materials, as for any lattice structure, are generally anisotropic $[18,22,83-85]$. This is directly related to the symmetry type exhibited by the lattice, cubic symmetry in the present cases. By means of finite element analysis combined with computational homogenization, the anisotropic response of the regular and inverse hexaround cells will be explored.

\section{Computational Strategy}

Sample step files were generated using CATIA v5R20 (Dassault Systèmes). The first step is defining a parametric line model of the cells. Lines are then transformed into struts by assigning them a circular thickness. Boolean intersection operation is used to circumscribe the cell inside a cubic volume of side $L$. Since the unit cells present a cubic symmetry, only a $1 / 8$ of the cell needs to be modeled. The entire cell is reconstituted subsequently by applying symmetry directly onto the mesh.

CAD files (STEP format) are meshed (see Fig. 4) using GMSH software [86] with tetrahedral quadratic elements (C3D10). A maximal mesh size of $0.05 \mathrm{~mm}$ is applied, ensuring the presence of at least five elements in the thickness of struts, even for the smallest volume fraction. This mesh size have been validated through convergence analysis, ensuring an error inferior to $0.5 \%$, compared with a converged mesh (see Appendix B).

Those mesh files can then be imported into Z-set finite element package $^{2}$ and converted into the native mesh format. Finally, sets of corresponding nodes are created on the outer boundary of the mesh to implement periodic boundary conditions later on.

A total of 70 parametric configurations, i.e., virtual samples, are generated for computing the effective elastic properties. Computation time is dependent on the mesh size, which is directly linked to the relative density of the cell. The data presented in Table 1 correspond to single threading on a Intel Xeon E5-2640 v4 CPU running at $2.4 \mathrm{GHz}$. The computation time corresponds to the

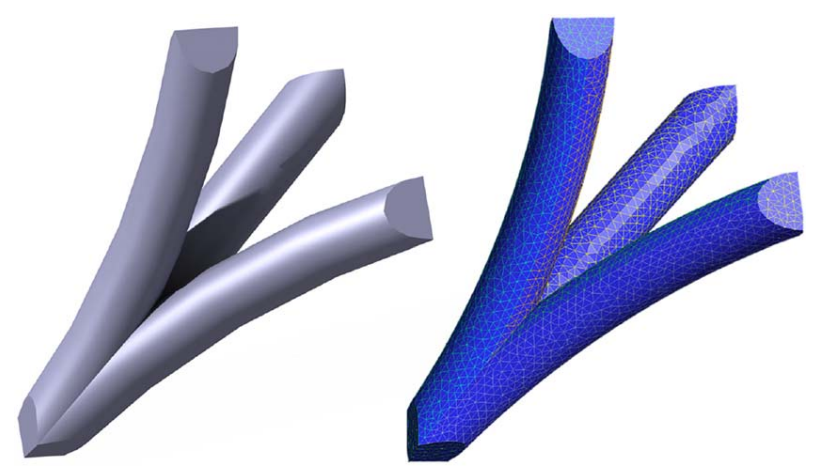

Fig. 4 1/8 hexaround cell CAD (left) and its corresponding mesh (right)

Table 1 Computation time and mesh size depending on the cell density

\begin{tabular}{lccc}
\hline \hline$\rho^{*}$ & Nb of elements & Memory (MB) & Comp. time (s) \\
\hline 0.05 & $52 \mathrm{k}$ & 1700 & 887 \\
0.10 & $97 \mathrm{k}$ & 4700 & 1620 \\
0.15 & $157 \mathrm{k}$ & 9800 & 2568 \\
0.20 & $207 \mathrm{k}$ & 16,100 & 3415 \\
0.25 & $270 \mathrm{k}$ & 26,300 & 4397 \\
\hline \hline
\end{tabular}

${ }^{2}$ http://www.zset-software.com time spent to identify the full elastic moduli tensor for one unit cell configuration. For instance, the computation of a 0.05 relative density configuration requires $695 \mathrm{~s}$ to import the mesh, and $32 \mathrm{~s}$ per computation (six computations are needed).

\section{Computational Homogenization}

Computational homogenization identifies the effective properties of a heterogeneous medium, by computing the volume-averaged response under a physical stimulus over a virtual sample that is considered as a representative volume element (RVE). In the present work, we rely on full-field finite element mechanical analysis using periodic boundary conditions. For the case of elastic periodic lattices, the RVE consists of the periodic unit cell defined by its periodicity vectors $\left(\underline{X_{i}}\right.$ with $i=3$ for three-dimensional structures, as shown in Fig. 1).

Periodic boundary conditions are considered for studying the behavior of auxetic lattice structures as they are found to be optimal for determining the effective properties of an infinite medium made of periodic unit cells $[87,88]$. The application of periodic boundary conditions allows for the identification of the overall response of architectured materials with vanishing boundary layer effects, which is very useful if one wants to use the identified response as the constitutive behavior of a continuum element in a larger scale simulations.

Periodic boundary conditions are enforced by multiple-point constraints applied on sets of corresponding mesh nodes that are separated by periodicity vectors, as depicted in Fig. 1, or by a linear combination of periodicity vectors with integer coefficients. Another main interest of computational homogenization resides in obtaining an equivalent constitutive model that can be implemented in a finite element analysis, saving extensive computation time by avoiding to explicitly represent and account for the underlying microstructure. This strategy is commonly used for architectured materials [5], allowing for fast computation of their effective properties. In this work, linear elastic properties of auxetics are investigated first, before extending the study to elastoplasticity.

Considering the small deformation hypothesis, constitutive relations are expressed locally in a linear elasticity framework using the generalized Hooke law:

$$
\underset{\sim}{\boldsymbol{\sigma}}(\underline{x})=\underset{\sim}{\boldsymbol{c}}(\underline{x}): \underset{\sim}{\boldsymbol{\varepsilon}}(\underline{x})
$$

where $\boldsymbol{\sigma}$ is the second-order symmetric Cauchy stress tensor, $\boldsymbol{\varepsilon}$ is the second-order symmetric engineering strain tensor, and $\boldsymbol{c} \sim$ is the fourth-order positive definite tensor of elastic moduli. Considering a volume element $V$, the macroscopic stress and strain tensors $\boldsymbol{\Sigma}$ and $\underset{\sim}{\boldsymbol{E}}$ are defined by the spatial averages over $V$ of local stress $\underset{\sim}{\boldsymbol{\sigma}}$ and strain $\underset{\sim}{\boldsymbol{\varepsilon}}$ fields.

$$
\begin{aligned}
& \underset{\sim}{\boldsymbol{\Sigma}}:=\langle\underset{\sim}{\boldsymbol{\sigma}}\rangle=\frac{1}{V} \int_{V} \underset{\sim}{\boldsymbol{\sigma}} d V \\
& \underset{\sim}{\boldsymbol{E}}:=\langle\underset{\sim}{\boldsymbol{\varepsilon}}\rangle=\frac{1}{V} \int_{V} \underset{\sim}{\boldsymbol{\varepsilon}} d V
\end{aligned}
$$

Equations. (8)-(10) yield the tensor of effective elastic moduli $\underset{\approx}{\boldsymbol{C}}$

$$
\underset{\sim}{\boldsymbol{\Sigma}}=\underset{\approx}{\boldsymbol{C}}: \underset{\sim}{\boldsymbol{E}}
$$

For periodic boundary conditions, the displacement field $u$ can be dissociated into a part given by the macroscopic strain tensor $\boldsymbol{E}$ and a periodic fluctuation field $v$ for any material point $x$ of $\tilde{V}$, such that

$$
\underline{u}=\underset{\sim}{\boldsymbol{E}} \cdot \underline{x}+\underline{v} \quad \forall \underline{x} \in V
$$


where $v$ is the periodic fluctuations vector, i.e., taking the same value for two homologous points $\underline{x}^{+}$and $x^{-}$of $\partial V$. Furthermore, the traction vector $\underline{t}=\underset{\sim}{\sigma} \cdot \underline{n}$ fulfills antiperiodic conditions such that

$$
\begin{gathered}
{\underset{\sim}{\boldsymbol{\sigma}}}^{+} \cdot \underline{n}^{+}+\underline{\sim}^{-} \cdot \underline{n}^{-}=\underline{0} \\
\underline{v}^{+}-\underline{v}^{-}=\underline{0}
\end{gathered}
$$

The elastic moduli tensor $\boldsymbol{C}$ can then be fully identified by applying successively six fundamental macroscopic loading, three pure extensions, and three pure shears. Although the hexaround and inverse hexaround cells present cubic symmetry, six independent computations are run since the developed methodology is generic and automated. The elastic moduli tensor is identified by applying the macroscopic strain $\underset{\sim}{\boldsymbol{E}}$ by using Voigt's notation:

$$
\left[\begin{array}{c}
\Sigma_{11} \\
\Sigma_{22} \\
\Sigma_{33} \\
\Sigma_{23} \\
\Sigma_{31} \\
\Sigma_{12}
\end{array}\right]=\left[\begin{array}{cccccc}
C_{11} & C_{12} & C_{12} & 0 & 0 & 0 \\
C_{12} & C_{11} & C_{12} & 0 & 0 & 0 \\
C_{12} & C_{12} & C_{11} & 0 & 0 & 0 \\
0 & 0 & 0 & C_{44} & 0 & 0 \\
0 & 0 & 0 & 0 & C_{44} & 0 \\
0 & 0 & 0 & 0 & 0 & C_{44}
\end{array}\right]\left[\begin{array}{c}
E_{11} \\
E_{22} \\
E_{33} \\
2 E_{23} \\
2 E_{31} \\
2 E_{12}
\end{array}\right]
$$

Linear relations thus appear between macroscopic stress and strain and can readily be used to build up effective elastic moduli tensors for a given microstructure. The formalism is similar for any linear property, e.g., thermal conductivity. To implement periodic boundary conditions, we rely on the following specific finite element approach, i.e., adding global degrees of freedom (DOFs) shared by all elements. These DOFs correspond to the macroscopic strain components $E_{i j}$ for displacements $v_{i}$ in addition to the classical nodal DOFs.

The finite element problem left to solve concerns the homogeneous strain tensor $E_{i j}$ and its dual $R_{i j}$, which corresponds to the macroscopic reaction stress. Prescribing $E_{i j}$ corresponds to the macroscopic strain approach, while prescribing $R_{i j}$ leads to the macroscopic stress approach. In that way, mixed macroscopic problems, e.g., tension, can be solved with periodic boundary conditions.

We note that the effective elastic properties are varying with loading orientations, thus we should rather refer to elastic modulus functions or Poisson's ratio functions. Nevertheless, for the sake of simplicity and comparison with most of the literature on auxetics, we will use the conventional denomination of Young's modulus and Poisson's ratio, although these are, by definition, isotropic.

Computing the elastic moduli tensor $\boldsymbol{C}$ is done for every set of parameters considered for both unit cellis. The resulting data can be represented spatially by expressing elastic properties within a particular coordinates framework. By using the Euler-Bunge [89] angles $\phi, \theta$, and $\psi$, as shown in Fig. 5, let us define the three orthogonal vectors $\underline{l}, \underline{m}$, and $\underline{n}$, such that

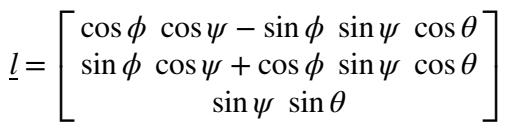

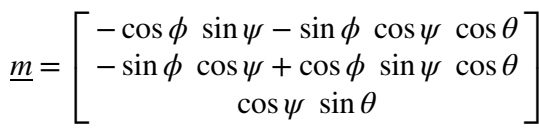

$$
\begin{aligned}
& \underline{n}=\left[\begin{array}{c}
\sin \phi \sin \theta \\
-\cos \phi \sin \theta \\
\cos \theta
\end{array}\right]
\end{aligned}
$$

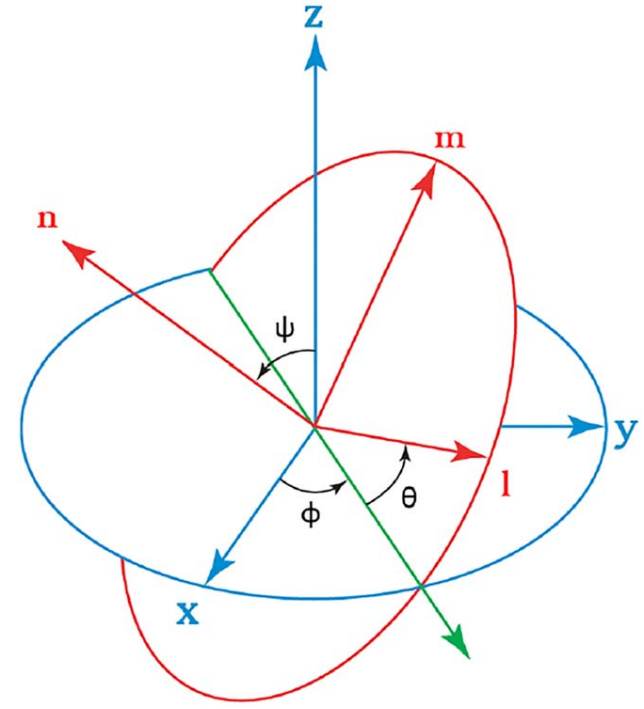

Fig. 5 Euler-Bunge angles

Using the macroscopic stress $\underset{\sim}{\boldsymbol{\Sigma}}(\phi, \theta, \psi)$ and strain $\underset{\sim}{\boldsymbol{E}}(\phi, \theta, \psi)$ tensors, effective Young's modulus $E(l)$ and Poisson's ratio $\nu^{*}(l$, $m)$ are defined as follows:

$$
\begin{gathered}
E=\frac{\underline{l} \cdot \underset{\sim}{\underset{\sim}{\boldsymbol{\Sigma}} \cdot \underline{l}}}{\underline{\underline{l}} \cdot \underset{\sim}{\boldsymbol{E}} \cdot \underline{l}} \\
\nu^{*}=\frac{\underline{m} \cdot \underset{\sim}{\boldsymbol{E}} \cdot \underline{m}}{\underline{\underline{l}} \cdot \underset{\sim}{\boldsymbol{E}} \cdot \underline{l}}
\end{gathered}
$$

To compare the different geometrical configurations with each other, normalized elastic properties need to be defined, based on the properties of the constitutive material, considered here to be a Ti-6Al-4V titanium alloy $\left(E_{0}=97 \mathrm{GPa}, \nu_{0}=0.3\right)$, and the relative density $\rho^{*}$ of each configuration. The normalized Young modulus is defined as follows:

$$
E^{*}=\frac{1}{E_{0} \rho^{*}} E
$$

The relative density of a cell can be defined as the actual volume of the cell $V_{\text {lattice }}$ divided by the cubic volume circumscribed to the cell $\left(V_{\text {cubic }}=L^{3}\right.$ ), as depicted in Fig. 6, yielding the following definition:

$$
\rho^{*}=\frac{V_{\text {lattice }}}{V_{\text {cubic }}}
$$

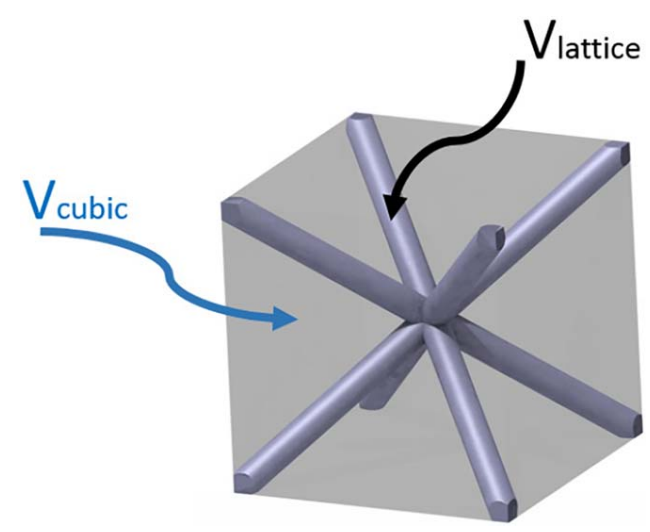

Fig. 6 Definition of $V_{\text {lattice }}$ and $V_{\text {cubic }}$ 


\section{Results}

Effective elastic properties have been computed for both unit cells, considering all combinations of parameters, for $L=3 \mathrm{~mm}$, $a \in[0.10 ; 0.75] \mathrm{mm}$, and $\rho^{*} \in[0.05 ; 0.25]$, which corresponds to the variation of $D$, the strut diameter.

5.1 Hexaround Cell. As mentioned in Sec. 2, it is expected that a pre-buckled cell will exhibit auxetic properties. To investigate the pre-buckling effect on effective elastic properties, parameter $a$ is varied. Effect of the relative density $\rho^{*}$ is also explored by adjusting the radius of the struts. Figure 7 illustrates the fact that struts are merging at the corners of the hexaround cell. For an equivalent density, longer struts corresponding to higher values of $a$ require a lower radius. This trend persists until $a=0.5 \mathrm{~mm}$, before reversing for higher values of $a$, as a consequence of struts merging in the corner of the cell.

5.1.1 Young's Modulus. For all configurations investigated, the elastic moduli tensor $\boldsymbol{C}$ is computed. These moduli are exploited to evaluate the dependeñce of the Normalized Young's modulus and its anisotropy. For the sake of conciseness, the data are only represented as three-dimensional maps for extreme values for parameters $a$ and $\rho^{*}$, as shown in Fig. 8. On the one hand, this figure confirms that increasing the relative density of the lattice, i.e., the strut diameter, increases its stiffness and decreases the elastic anisotropy. On the other hand, increasing the buckling amplitude $a$ not only results in decreasing the elastic modulus in every direction, but also increases anisotropy. Pre-buckling consequences on stiffness are expected: since the struts are not straight

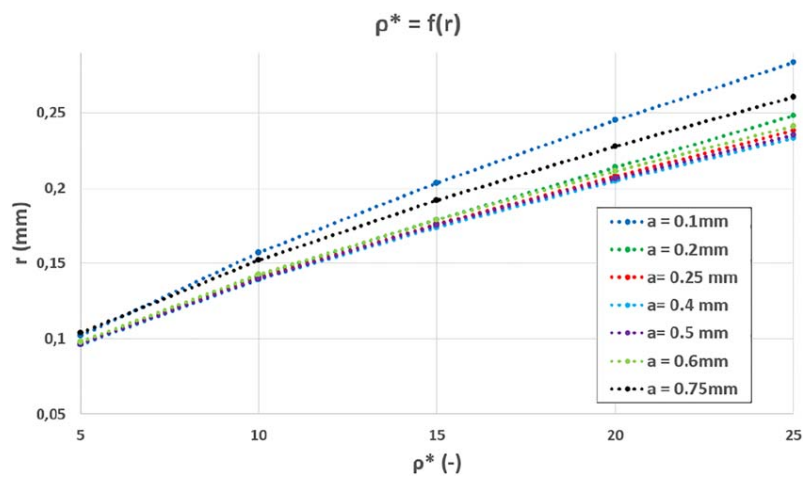

Fig. 7 Effect of the strut radius on the relative density for the hexaround cell

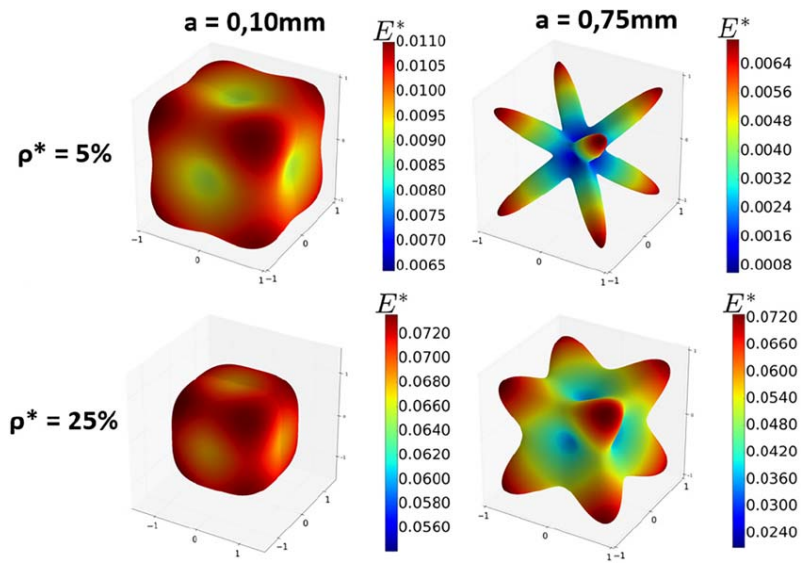

Fig. 8 3D maps of normalized Young's modulus for various relative density $\rho^{\star}$ and pre-buckling amplitude $a$ for the hexaround cell anymore and not directly oriented along the applied loading, stiffness is inevitably reduced. Figure 8 also indicates that for all configurations explored, the direction maximizing the Young Modulus is $\langle 111\rangle$, by analogy with Miller indices in crystallography, i.e., all equivalent directions corresponding to the diagonal of the cubic cell.

The maximal values of normalized Young's modulus, i.e., along $\langle 111\rangle$, have been computed for all configurations, as shown in Fig. 9. From this figure, it yields that the maximal stiffness of the hexaround cell increases monotonously while increasing relative density $\rho^{*}$. On the contrary, the evolution of the maximal stiffness is nonmonotonous with $a$ : first, $E^{*}$ decreases while increasing $a$, but for $a=0.75 \mathrm{~mm}$, stiffness increases again due to the struts merging at the corner of the cell. Merging of the struts also takes place while increasing relative density, which explains the convergence of stiffness observed for $\rho^{*}=0.25$. These last two remarks justify the use of full-field finite element simulation to study the behavior of auxetic lattices, as reasonably simple analytical models would not be able to account for such geometrical effects.

5.1.2 Poisson's Ratio. Effective Poisson's ratio is computed in the $(l, m)$ plane, which includes the direction $l$ of applied load. For the sake of clarity, the effective Poisson ratio $\nu^{*}$ is represented within one plane, for specific orientations, in Fig. 10, for different values of $a$ but density fixed at $\rho^{*}=0.05$. As a matter of fact, the auxetic character of the hexaround lattice is due to its re-entrant topology, which is less effective for higher densities. Computations

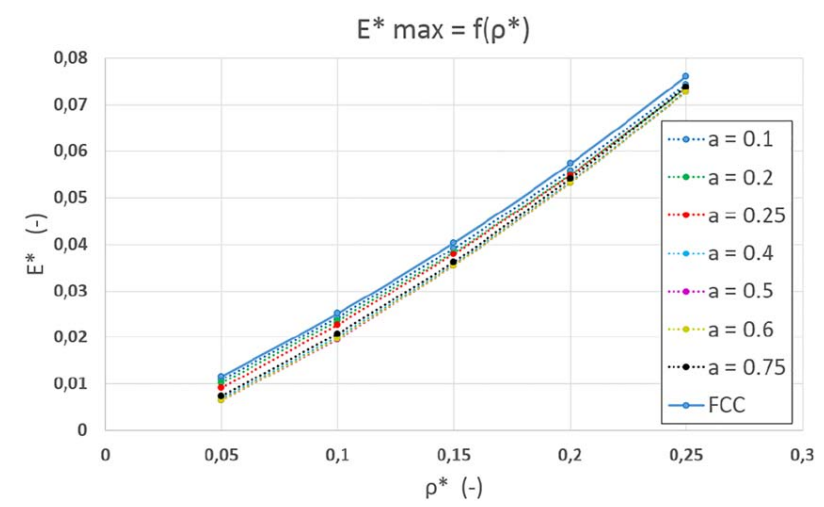

Fig. 9 Hexaround maximal effective Young's modulus with respect to relative density $\rho^{\star}$ and pre-buckling amplitude a

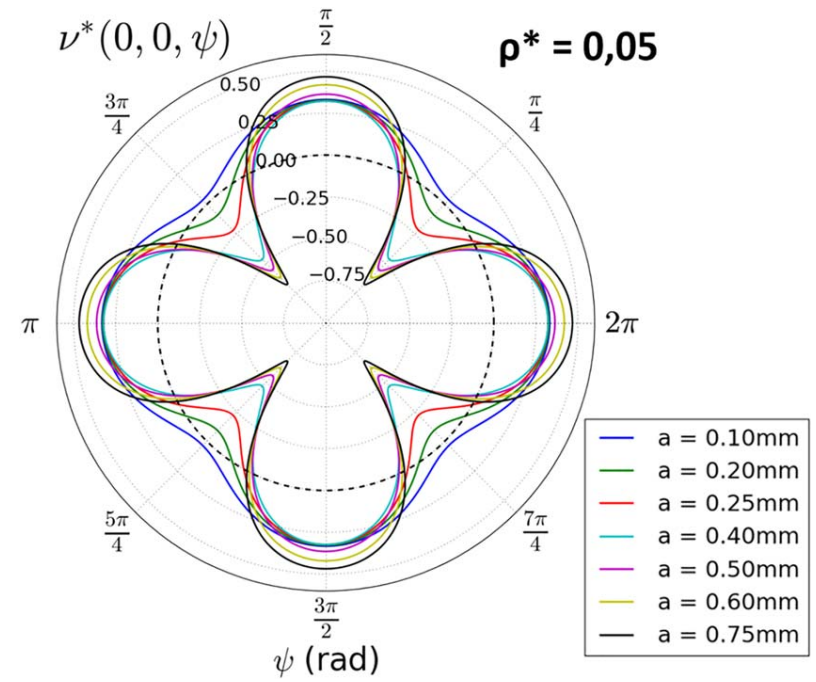

Fig. 10 Hexaround Poisson's ratio within the (100) plane, for various $a$ and $\rho^{\star}=0.05$ 
have showed that the minimal Poisson ratio is to be found within the (100) plane, following the crystallographic analogy. From Fig. 10, it can be observed that auxetic behavior is achieved through prebuckled design for a certain angular range, i.e., $[\pi / 8,3 \pi / 8] \pm \pi / 2$. The influence of the pre-buckling distance $a$ is critical: increasing $a$ results in a lower negative Poisson ratio at $\psi=\pi / 4$, and a higher positive one at $\psi=0[2 \pi]$, hence resulting in a higher anisotropy for the Poisson ratio. For the configuration with $a=0.1 \mathrm{~mm}$, no auxetic behavior is observed, while the effect is maximal for $a=$ $0.75 \mathrm{~mm}$, with $\nu^{*}=-0.73$. The minimal Poisson ratio is obtained for the $(0,0, \pi / 4 \pm \pi / 2)$ direction within the (100) plan, which corresponds to the $\langle 110\rangle$ equivalent directions.

The most negative values of Poisson's ratio, i.e., along the $\langle 110\rangle$ equivalent directions, have been computed for every parametric configurations of the hexaround cell and plotted in Fig. 11. The increase of relative density results in an increase of the Poisson ratio. As mentioned earlier, this was expected due to the vanishing of the lattice re-entrant topological character with increasing density.

5.2 Inverse Hexaround Cell. For the hexaround cell, effective elastic properties are investigated depending on parameters $a$ and $\rho^{*}$. Also, the same type of strut merging phenomenon is evidenced for higher density inverse hexaround lattice structures, through a larger strut radius, as represented in Fig. 12.

5.2.1 Young's Modulus. In the same fashion for the hexaround cell analysis, the normalized Young modulus is obtained for any spatial orientation for the inverse hexaround cell based on the tensor of elastic moduli. Again, for the sake of clarity and conciseness, only the data obtained for extreme parameters values have been plotted as 3D maps on Fig. 13, since intermediary values follow a monotonous trend. From this figure, it can be seen that increasing the relative density results in increasing stiffness and decreasing anisotropy, for the hexaround lattice. Also, the effective

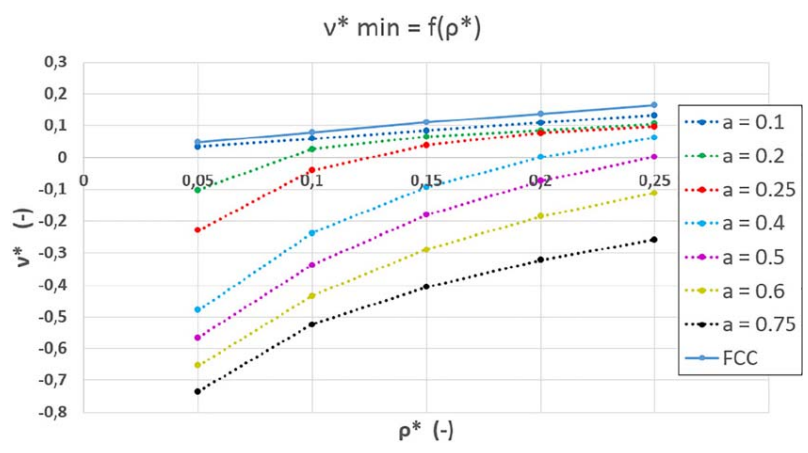

Fig. 11 Most negative Poisson's ratio values for the hexaround lattice with respect to $a$ and $\rho^{*}$

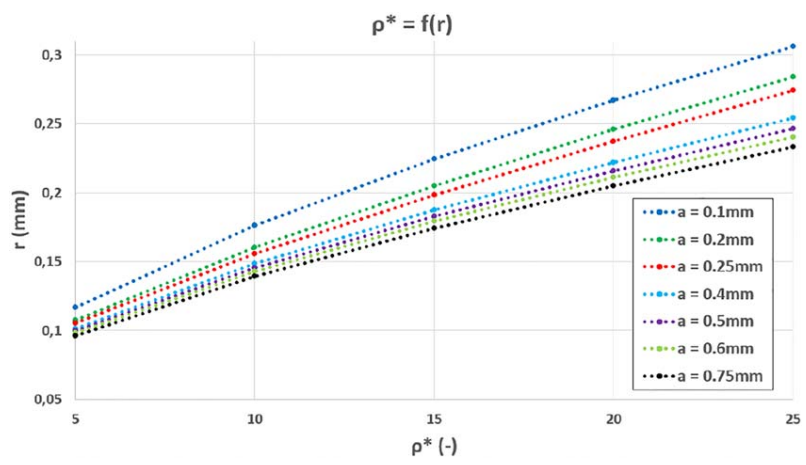

Fig. 12 Effect of the strut radius on the relative density for the inverse hexaround cell

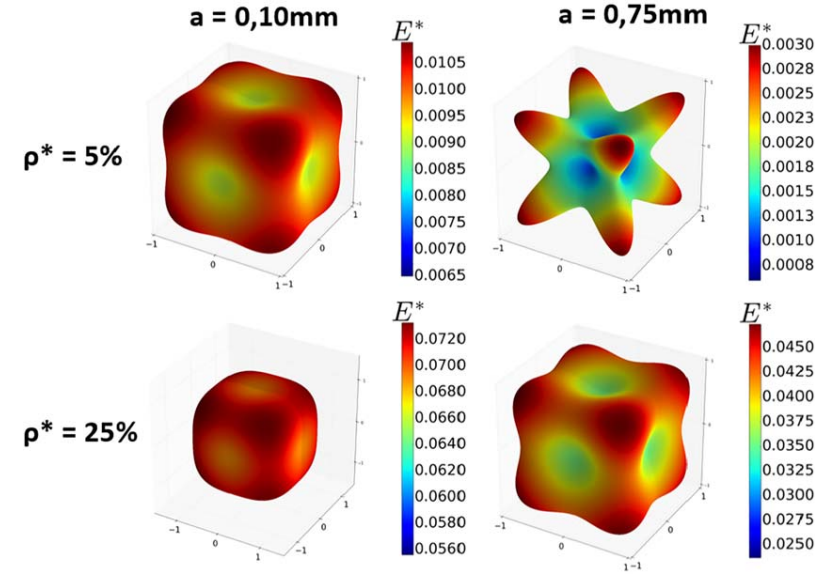

Fig. 13 3D maps of normalized Young's modulus for various relative density $\rho^{\star}$ and pre-buckling amplitude $a$ for the inverse hexaround cell

elastic modulus is inversely proportional to the buckling amplitude $a$, without exception. In Fig. 13, it can also be seen that the maximal effective Young modulus is found along the $\langle 111\rangle$ equivalent directions, similar to the hexaround cell.

For the previous lattice, the maximal effective Young modulus values, i.e., along the $\langle 111\rangle$ directions, are plotted against the relative density $\rho^{*}$ and buckling amplitude $a$ in Fig. 14 for the inverse hexaround cell. Figure 14 shows that maximal stiffness increases monotonously with relative density, while evolving inversely with buckling amplitude $a$. It appears that the effective Young modulus values do not converge toward a single value, mainly because the strut merging effect remains marginal with the inverse hexaround topology.

5.2.2 Poisson's Ratio. For the hexaround lattice, the effective Poisson ratio is computed in the $(l, m)$ plane, which includes the direction $\underline{l}$ of applied load. The Poisson ratio $\nu^{*}$ is represented within one plane, for specific orientations, in Fig. 15, for various values of $a$ but density fixed at $\rho^{*}=0.05$. Again, the auxetic effect is more pronounced for lower density. Also, for the hexaround cell, computations revealed that the minimal Poisson ratio is to be found within the (100) plane. Exploiting Fig. 15, it can be seen that auxetic behavior is achieved for the same angular range as before, i.e., $[\pi / 8,3 \pi / 8] \pm \pi / 2$. The same trend can be observed for the effect of pre-buckling amplitude $a$ : increasing $a$ yields a higher anisotropy, with a more negative Poisson ratio, i.e., a more pronounced auxetic effect, while not changing the maximum positive Poisson ratio value. A higher value of $a$ is needed for the inverse hexaround lattice to exhibit an auxetic character. The auxetic effect is maximal for $a=0.75 \mathrm{~mm}$, with

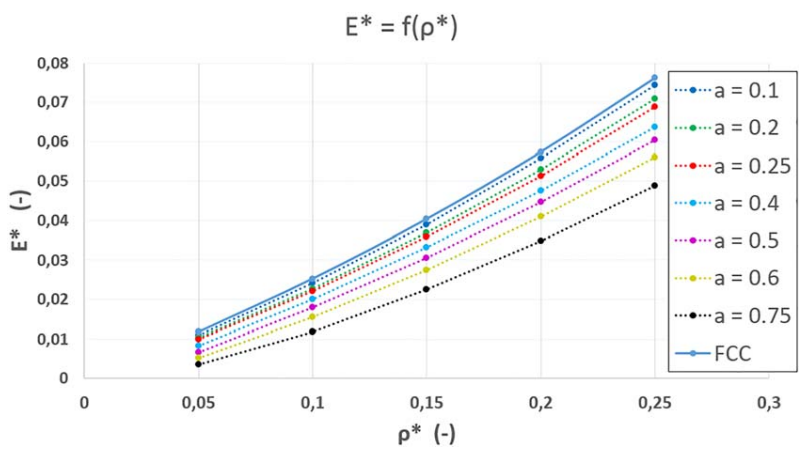

Fig. 14 Inverse hexaround maximal effective Young's modulus with respect to relative density $\rho^{\star}$ and pre-buckling amplitude a 


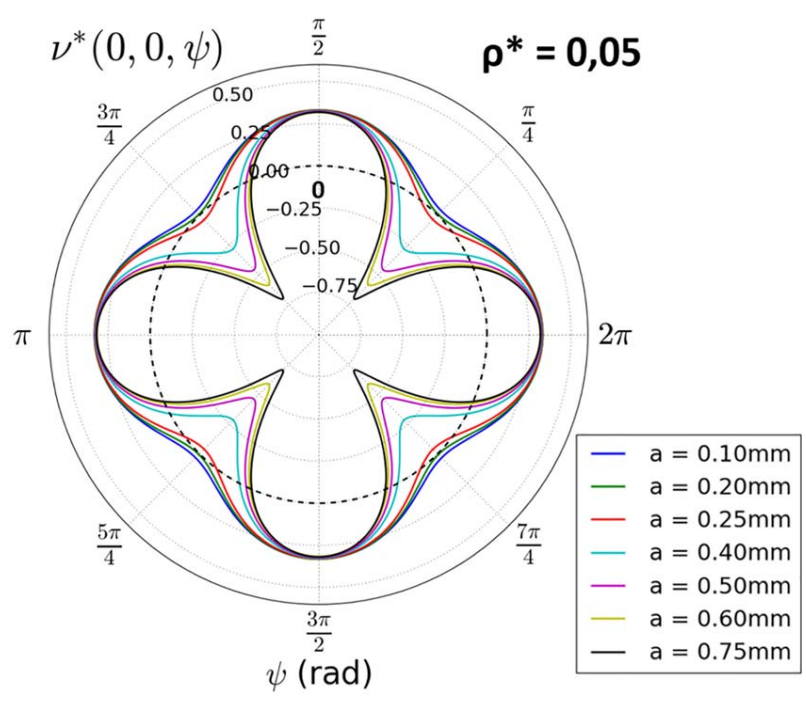

Fig. 15 Inverse hexaround Poisson's ratio within the (100) plane, for various a and $\rho^{\star}=0.05$

$\nu^{*}=-0.70$, along the $(0,0, \pi / 4 \pm \pi / 2)$ direction within the (100) plane, which corresponds to the $\langle 110\rangle$ equivalent directions, for the hexaround lattice.

The most negative Poisson ratio values have been computed for each parametric configurations of the inverse hexaround cell and plotted in Fig. 16. As expected, increasing relative density increases $\nu^{*}$.

\section{Extension to Elastoplasticity}

Studies have suggested that auxetic behavior could improve crashworthiness [43-46]. In this section, the influence of the loading direction and relative density on the mechanical energy dissipation through plastic work will be explored in the case of an elastoplastic constitutive material. It has been shown in Sec. 5 that various mechanical responses can be obtained for specific orientations in space due to anisotropy of the elastic behavior in lattice structures. Both unit cells exhibit minimal effective Poisson's ratio when loaded along $\langle 110\rangle$ equivalent directions and maximal Young's modulus along $\langle 111\rangle$. To investigate the relation between elastic properties, elastic anisotropy, and mechanical energy dissipation, a series of computational experiments are conducted through finite element simulation. The energy dissipation is computed as the total plastic work up to $5 \%$ of macroscopic deformation under uniaxial compression. For the sake of simplicity, damage is not considered in the simulation. Thanks to the periodic

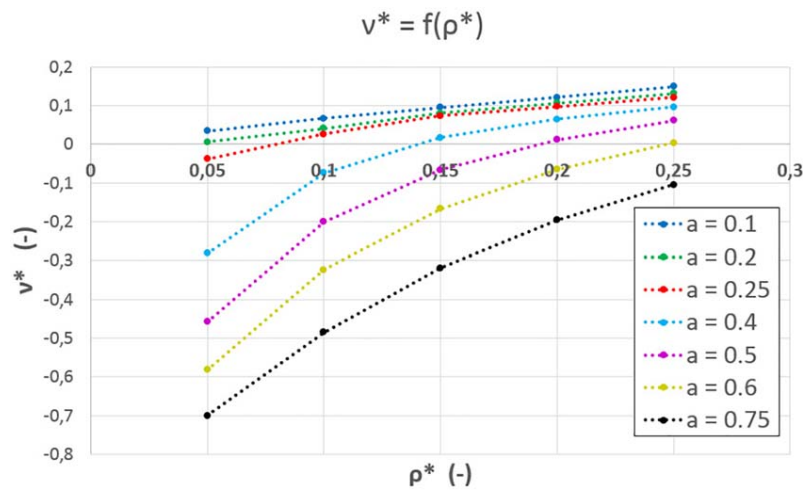

Fig. 16 Most negative Poisson's ratio values for the inverse hexaround lattice with respect to a and $\rho^{*}$ element type introduced in Sec. 4, the compression state is obtained by applying a macroscopic stress tensor $\underset{\sim}{\boldsymbol{\Sigma}}$, such that

$$
\underset{\sim}{\boldsymbol{\Sigma}}=\left[\begin{array}{lll}
1 & 0 & 0 \\
0 & 0 & 0 \\
0 & 0 & 0
\end{array}\right]
$$

The dissipated mechanical energy $\mathcal{E}_{\mathrm{d}}$ during the deformation of an elastoplastic material is defined as the integral of the stressplastic strain curve evaluated up to a final strain, noted $\varepsilon_{f}$, as in Eq. (24), hence corresponding to the total plastic work for a monotonic loading. It is obtained as a local post-processing on each integration point within the simulation.

$$
\mathcal{E}_{\mathrm{d}}=\int_{0}^{\varepsilon_{f}} \sigma d \varepsilon^{p}
$$

6.1 Material Properties. The mechanical behavior of the constitutive material is extended to elastoplasticity. Let us consider a simple yield function:

$$
f(\boldsymbol{\sigma})=\boldsymbol{\sigma}^{\mathrm{eq}}-R
$$

with the von Mises equivalent stress $\sigma^{\mathrm{eq}}$ such that

$$
\boldsymbol{\sigma}^{\mathrm{eq}}=\sqrt{\frac{3}{2}{\underset{\sim}{\boldsymbol{\sigma}}}^{\mathrm{dev}} \boldsymbol{\sigma}_{\sim}^{\mathrm{dev}}}
$$

where $\boldsymbol{\sigma}^{\mathrm{dev}}$ is the deviatoric part of the stress tensor. In addition, an isotropic nonlinear hardening model is adopted, as shown in Eq. (27):

$$
R=R_{0}+\sum_{i}^{2} Q_{i}\left(1-e^{-b_{i} p}\right)
$$

The constitutive material considered is a Ti-6Al-4V titanium alloy, with elastoplastic behavior, i.e., strain rate independent, and material modeling parameters identified from tensile experiments available in the literature [90]: $E=97 \mathrm{GPa}, \nu=0.3, \sigma_{Y}=$ $759 \mathrm{MPa}, Q_{1}=331 \mathrm{MPa}, b_{1}=332, Q_{2}=259 \mathrm{MPa}$, and $b_{2}=5.8$.

6.2 Dissipated Energy Comparison. To compare the amount of dissipated energy with respect to the loading orientation, three orientations have been considered for the lattices: principal orientation [100], minimal Poisson's ratio [110], and maximal Young's modulus [111] orientations. Also, choice has been made to compare the dissipated energy for both cells at the configuration $a=0.6 \mathrm{~mm}$, which exhibits auxetic behavior for all $\rho^{*}$, only with varying relative density.

6.2.1 Hexaround Cell. Numerical compression tests along three orientations have been done, keeping same parameter $a$ and varying relative density. The dissipated energy has been measured following Eq. (24) up to $10 \%$ plastic strain and plotted with respect to relative density in Fig. 17. The value of $10 \%$ plastic strain has been chosen for being representative of the level of deformation that could be sustained by the lattice in realistic applications, yielding the local strain level up to $25-30 \%$, which would induce failure of the constitutive material, i.e., Ti-6Al-4V. From this figure, it can be seen that energy dissipation is following a power law dependence, at least between $\rho^{*}=0.05$ and 0.25 , for all three loading directions. The most efficient orientation for the hexaround lattice to dissipate energy through plastic work appears to be along the [111] direction, suggesting that Young's modulus is a critical parameter for energy dissipation through plastic work. Direction [110] also dissipates more than the principal [100] cubic orientation. 


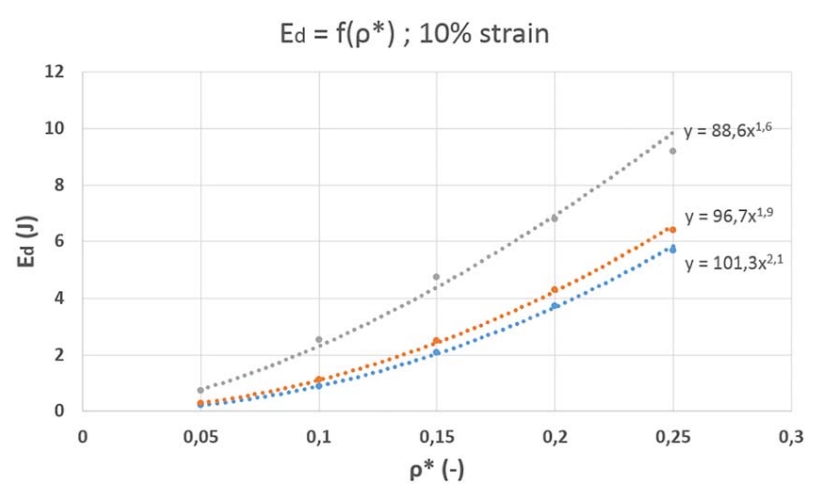

Fig. 17 Energy dissipated by the hexaround unit cell during the compression testing through three different orientations, with respect to the relative density

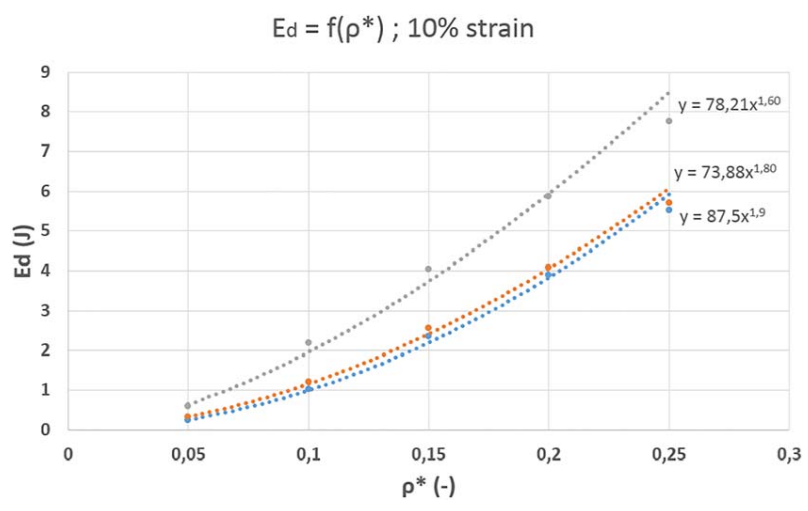

Fig. 18 Energy dissipated by the inverse hexaround unit cell during the compression testing through three different orientations, with respect to the relative density

6.2.2 Inverse Hexaround Cell. For the hexaround lattice, the dissipated energy has been measured up to $10 \%$ plastic strain and plotted against relative density in Fig. 18. The energy dissipation evolves to the relative density, following a power law relation on the investigated range of densities, and this for all three loading directions. Although the most efficient orientation is still along [111], there is a competition between the [110] and [100] directions: by extrapolating the results for higher densities, it seems that the minimal Poisson's ratio direction would be less efficient, although stiffer than [100].

\section{Discussion}

Cellular auxetics appear as interesting candidates due to densification under indentation or compression loading, and all things equal otherwise, auxetics should behave better under impact, for instance when used as sandwich-core material to be mainly loaded in shear, due to the high relative shear modulus of linear isotropic auxetics. Thirty-five years of auxetics research have shown that auxetics, either deterministic or stochastic, are bendingdominated architectures, hence yielding a very soft response with respect to classical stretch-dominated lattices or shell-lattices [69]. This soft elastic response could result in a more progressive behavior in the elastoplastic regime and potentially anisotropy that could be exploited to create bespoke materials with tailored mechanical behavior. Although auxetic behavior has been effectively obtained through pre-buckled design, as shown in Figs. 10 and 15 , one can notice that the regular and inverse hexaround lattices are not as fully auxetic, i.e., auxetic in every direction, as for most auxetic lattices available in the literature. Only specific angular domains exhibit effective negative Poisson's ratio. It
Table 2 Comparison for effective mechanical properties between hexatruss, regular, and inverse hexaround cells for a compression test along the [111] direction up to $10 \%$ of plastic strain

\begin{tabular}{lcccc}
\hline \hline Cell & $\rho^{*}$ & $\mathrm{E}^{*} \max$ & $\nu^{*} \min$ & $\mathcal{E}_{d}(\mathrm{~J})$ \\
\hline Hexaround & 0.10 & 0.020 & -0.435 & 2.53 \\
Inverse hexaround & 0.10 & 0.016 & -0.329 & 2.20 \\
Hexatruss & 0.10 & 0.014 & -0.533 & 1.80 \\
\hline \hline
\end{tabular}

suggests that auxeticity is related to both shape and amplitude of buckling. Amplitude of buckling is the main parameter driving the Poisson ratio value, followed by the relative density. Both parameters also have a strong influence on the effective Young modulus: the higher the buckling amplitude, the lower the modulus and the more negative the Poisson ratio. Hexaround and inverse hexaround unit cells exhibit similar effective elastic properties at the same buckling amplitude for low relative density, but the hexaround lattice exhibits both higher modulus and more pronounced auxeticity at high relative density compared with the inverse hexaround lattice. Also, no influence of relative density on Poisson's ratio was observed in Ref. [91], most likely due to a small range of investigation for the relative density. In the present work, computational results on a wider range, from $\rho^{*}=0.05$ to 0.25 , show that the auxetic behavior of lattices depends strongly on the relative density. This claim is supported by other results in the literature on buckling-induced auxeticity [81,82]. According to Figs. 13-16, it appears that the inverse hexaround lattice might be a better choice for the application-oriented auxetic design, in the sense that its effective elastic properties evolve monotonously with respect to the geometrical parameters $a$ and $\rho^{*}$, making it easier to draw design guidelines in comparison with the regular hexaround lattice.

Regarding applications, crashworthiness is a key performance expected from architectured cellular materials such as auxetic lattices. By simulating the compression of both unit cells along three different orientations ([100], [110], and [111]), one can observe from Figs. 17 and 18 that both the regular and inverse hexaround lattices exhibit the highest plastic work dissipation along the [111] loading direction, which is also the direction of maximal effective stiffness. This result is contradictory to Ref. [45], which reports in a quasi-2D orthotropic case, that the energy absorption is maximal for auxetic configurations that are not the stiffest. Although three dimensional, the lattices structures introduced in the present work exhibit a higher degree of symmetry, therefore leading to cubic anisotropy, which drives the mechanical response of the lattice $[40,92,93]$. To conclude on the crashworthiness of the proposed lattices, an additional comparison was made with the hexatruss cell [22], also exhibiting a re-entrant topology, for the same buckling amplitude $a=0.6 \mathrm{~mm}$ and relative density $\rho^{*}=0.1$, by compressing the cells along the [111] direction up to $5 \%$ of plastic strain. The data are listed in Table 2. It can be seen from Table 2 that the hexatruss lattice displays not only a more negative Poisson ratio than other cells but also a lower maximal normalized Young modulus and a lower dissipated energy up to $10 \%$ plastic strain. This observation is consistent with the one made for the regular and inverse hexaround cells: a higher energy dissipation is obtained for 3D elastoplastic auxetic lattices loaded along the direction of maximum effective stiffness. Finally, it can be noted that the straightness of constitutive struts and sharps angles of the hexatruss design lead to stress concentrations and premature localized plasticity.

\section{Conclusion and Perspectives}

In this study, two new auxetic lattice designs have been proposed based on the pre-buckled topology. The effective mechanical properties of the lattices have been investigated through computational 
homogenization using periodic boundary conditions. These cells were conceived to exhibit negative Poisson's ratio due to the prebuckled design, which consists of curvilinear cylindrical struts, curved inward, i.e., toward the center of the cubic cell.

Computational homogenization results showed that auxetic behavior has effectively been achieved in this way. The influence of the geometrical parameters has been assessed, showing that the buckling amplitude $a$ has a critical impact on effective elastic properties, increasing the effective Poisson ratio and elastic anisotropy, as well as lowering the overall stiffness of the cells. On the other hand, increasing the relative density $\rho^{*}$ results in an increased stiffness, and a less and less auxetic as well as anisotropic lattice, as it could be expected by going from a thin lattice structure to a bulky porous material. The computational results also showed that the stiffest spatial orientation for both lattices are in the $\langle 111\rangle$ equivalent directions, while $\langle 110\rangle$ yield the most negative Poisson ratio.

Finally, an investigation about the influence of lattice orientation and its corresponding elastic properties on energy dissipation has been carried on the regular and inverse hexaround, as well as the hexatruss auxetic lattices, assuming elastoplasticity for the constitutive material with nonlinear isotropic hardening, using computational experiments, i.e., compression test simulation using finite elements with periodic boundary conditions in a finite deformation framework. Three different lattice orientations have been considered for the test: [100], a principal cubic direction; [111], a direction of maximum Young's modulus; and [110], a direction yielding the most negative Poisson ratio. Energy dissipation was measured as the total of plastic work up to $10 \%$ of plastic strain for different relative densities. The results showed that the direction of maximal effective stiffness, [111], dissipates the most for all three cells. These results may suggest that the effective Young modulus is the most important elastic properties for 3D auxetic lattices when aiming for energy dissipation through elastoplasticity, as the results are consistent for three lattice designs. The present work highlights the interest of investigating the influence of loading orientation and geometric parameters for crashworthiness performance evaluation, paving the way for tailored auxetic lattice materials designed for structural applications. Only monotonic loading has been considered in the present work; also, the current computational analysis does not account for any damage. To accurately estimate the dissipated energy, future investigations should incorporate damage, fatigue, and failure modeling.

\section{Acknowledgment}

F. Albertini and J. Dirrenberger acknowledge funding by the France-Australia Science Innovation Collaboration program. Financial support from the French Department of Higher Education, Research and Innovation is gratefully acknowledge for funding F. Albertini's Ph.D. scholarship. J. Dirrenberger acknowledge partial funding from the Agence Nationale de la Recherche through the ANR JCJC SCOLASTIC project (Grant No. 16-CE08-0009). A. Molotnikov would like to acknowledge the financial support from the Rod Rickards Fellowship from the Australian Academy of Science.

\footnotetext{
Nomenclature

$\mathrm{V}=$ volume

$\underset{\widetilde{c}}{\boldsymbol{c}}=$ microscopic elastic moduli tensor

$\underset{\widetilde{\widetilde{C}}}{\widetilde{\widetilde{E}}}=$ effective elastic moduli tensor

$\widetilde{\widetilde{E}}=$ macroscopic strain tensor

$E^{*}=$ normalized Young's modulus

$\underset{\sim}{\boldsymbol{\varepsilon}}=$ microscopic (local) strain tensor

$\nu^{*}=$ effective Poisson's ratio

$\rho^{*}=$ relative density

$\boldsymbol{\sigma}=$ microscopic (local) stress tensor

$\underset{\sim}{\widetilde{\Sigma}}=$ macroscopic stress tensor
}

\section{Appendix A: Struts Design}

The equations used to generate the lattice cell design rely on the geometrical description given in Fig. 19.

The Hexaround cell struts axes are obtained by fitting a circle through three points (P1, P2, and P3), C being its center. Then, only the arc starting at P1, finishing at $\mathrm{P} 3$, and passing through $\mathrm{P} 2$ is conserved. The radius of the circle has a direct influence on the buckling amplitude $a$ : the smaller the radius, the higher the buckling amplitude.

The equation for the corresponding circle is such that

$$
\left(x-\frac{L}{\sqrt{2}}\right)^{2}+\left(y-\left(\frac{a}{2}-\frac{L^{2}}{4 a}\right)\right)^{2}=r^{2}
$$

The inverse hexaround cell struts axis are obtained by fitting a circle through two points (P1 and P2), using the same radius than the hexaround circle. Only the arc starting at P1 and finishing at $\mathrm{P} 2$ is kept. This arc is then symmetrized with a plane of reflection. Influence of the radius on the buckling amplitude remains the same.

Equation of the circle that fit through P1 and P2 is as follows:

$$
x^{2}+(y-r)^{2}=r^{2}
$$
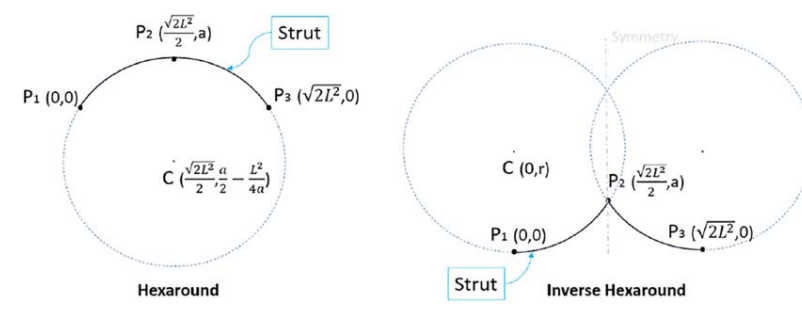

Fig. 19 Geometries used to define the struts of hexaround cell (left) and inverse hexaround cell (right)

\section{Appendix B: Mesh Convergence Analysis}

To ensure a mesh-independent response, a mesh sensitivity analysis has been performed, as shown in Fig. 20. From Fig. 20, it can be seen that an error lower than $0.5 \%$ has been measured on the [100] elastic modulus of the hexaround lattice in comparison with the maximal mesh density attainable with our current computational means, i.e., $271 \mathrm{k}$ elements, using $25 \mathrm{~GB}$ of RAM.

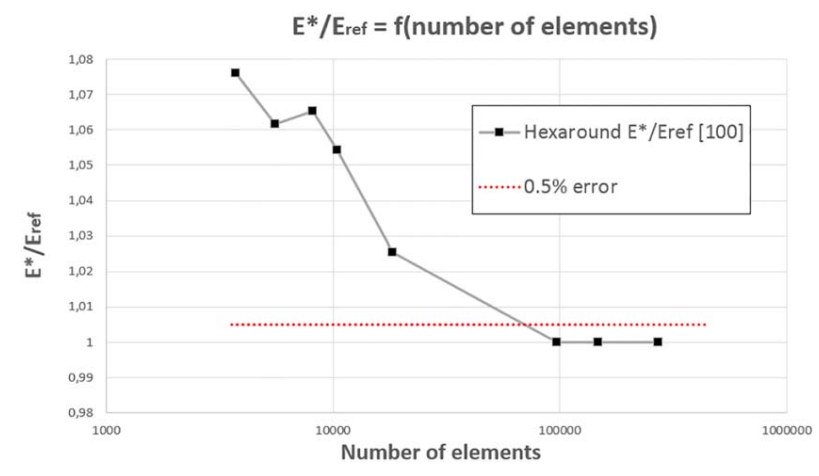

Fig. 20 Mesh sensitivity study for an hexaround cell with $a=$ $0.75 \mathrm{~mm}$ and $\rho^{\star}=0.10$. $E_{\text {ref }}$ is the converged normalized Young's modulus for a high mesh density (271k elements).

\section{References}

[1] Ashby, M. F., and Bréchet, Y., 2003, "Designing Hybrid Materials," Acta Mater., 51(19), pp. 5801-5821.

[2] Bouaziz, O., Bréchet, Y., and Embury, J. D., 2008, "Heterogeneous and Architectured Materials: A Possible Strategy for Design of Structural Materials," Adv. Eng. Mater., 10(1-2), pp. 24-36. 
[3] Bréchet, Y., and Embury, J. D., 2013, "Architectured Materials: Expanding Materials Space," Scr. Mater., 68(1), pp. 1-3.

[4] Dirrenberger, J., 2018, "Towards an Integrated Approach for the Development of Architectured Materials," Habilitation thesis, Sorbonne Université

[5] Dirrenberger, J., Forest, S., and Jeulin, D., 2019, "Computational Homogenization of Architectured Materials," Architectured Materials in Nature and Engineering, Y. Estrin, Y. Bréchet, J. Dunlop, and P. Fratzl, eds., Vol. 282 of Springer Series in Materials Science, Springer, New York, Chap. 4, pp. 89-139.

[6] Evans, K. E., Nkansah, M. A., Hutchinson, I. J., and Rogers, S. C., 1991 "Molecular Network Design," Nature, 353, p. 124.

[7] Herakovich, C. T., 1984, "Composite Laminates With Negative Through-theThickness Poisson's Ratios," J. Compos. Mater., 18(5), pp. 447-455.

[8] Almgren, R. F., 1985, “An Isotropic Three-Dimensional Structure With Poisson's Ratio=-1,” J. Elast., 15(4), pp. 427-430.

[9] Lakes, R. S., 1987, "Foam Structures With a Negative Poisson's Ratio," Science, 235(4792), pp. 1038-1040.

[10] Bathurst, R. J., and Rothenburg, L., 1988, "Note on a Random Isotropic Granular Material With Negative Poisson's Ratio," Int. J. Eng. Sci., 26(4), pp. 373-383.

[11] Caddock, B. D., and Evans, K. E., 1989, "Microporous Materials With Negative Poisson's Ratios: I. Microstructure and Mechanical Properties," J. Phys. D Appl. Phys., 22(12), pp. 1877-1882.

[12] Lakes, R. S., 1991, "Deformation Mechanisms in Negative Poisson's Ratio Materials: Structural Aspects,” J. Mater. Sci., 26(9), pp. 2287-2292.

[13] Milton, G. W., 1992, "Composite Materials With Poisson's Ratios Close to -1," J. Mech. Phys. Solids, 40(5), pp. 1105-1137.

[14] Prall, D., and Lakes, R. S., 1997, "Properties of a Chiral Honeycomb With a Poisson's Ratio of -1,” Int. J. Mech. Sci., 39(3), pp. 305-314.

[15] Yang, W., Li, Z.-M., Shi, W., Xie, B.-H., and Yang, M.-B., 2004, "Review on Auxetic Materials," J. Mater. Sci., 39(10), pp. 3269-3279.

[16] Hughes, T. P., Marmier, A., and Evans, K. E., 2010, "Auxetic Frameworks Inspired by Cubic Crystals,” Int. J. Solids Struct., 47(11-12), pp. 1469-1476.

[17] Alderson, A., Alderson, K. L., Attard, D., Evans, K. E., Gatt, R., Grima, J. N., Miller, W., Ravirala, N., Smith, C. W., and Zied, K., 2010, "Elastic Constant of 3-, 4- and 6-Connected Chiral and Anti-Chiral Honeycombs Subject to Uniaxial In-Plane Loading," Compos. Sci. Technol., 70(7), pp. 1042-1048.

[18] Dirrenberger, J., Forest, S., Jeulin, D., and Colin, C., 2011, "Homogenization of Periodic Auxetic Materials," Procedia Eng., 10, pp. 1847-1852.

[19] Pasternak, E., and Dyskin, A., 2012, "Materials and Structures With Macroscopic Negative Poisson's Ratio,” Int. J. Eng. Sci., 52, pp. 103-114.

[20] Alvarez Elipe, J. C., and Diaz Lantada, A., 2012, "Comparative Study of Auxetic Geometries by Means of Computer-Aided Design and Engineering," Smart Mater. Struct., 21, p. 105004

[21] Dirrenberger, J., Forest, S., and Jeulin, D., 2012, "Elastoplasticity of Auxetic Materials," Comput. Mater. Sci., 64, pp. 57-61.

[22] Dirrenberger, J., Forest, S., and Jeulin, D., 2013, "Effective Elastic Properties of Auxetic Microstructures: Anisotropy and Structural Applications," Int. J. Mech. Mater. Design, 9(1), pp. 21-33.

[23] Krasavin, V., and Krasavin, A., 2014, "Auxetic Properties of Cubic Metal Single Crystals,” Phys. Status Solidi (b), 251(11), pp. 2314-2320.

[24] Kaminakis, N., Drosopoulos, G., and Stavroulakis, G., 2015, "Design and Verification of Auxetic Microstructures Using Topology Optimization and Homogenization," Arch. Appl. Mech., 85(9), pp. 1289-1306.

[25] Körner, C., and Liebold-Ribeiro, Y., 2015, "A Systematic Approach to Identify Cellular Auxetic Materials," Smart Mater. Struct., 24(2), p. 025013.

[26] Saxena, K. K., Das, R., and Calius, E. P., 2016, "Three Decades of Auxetics Research- Materials With Negative Poisson's Ratio: A Review,” Adv. Eng. Mater., 18(11), pp. 1847-1870.

[27] Ren, X., Das, R., Tran, P., Ngo, T. D., and Xie, Y. M., 2018, "Auxetic Metamaterials and Structures: A Review," Smart Mater. Struct., 27(2), p. 023001

[28] Evans, K. E., 1991, "The Design of Doubly Curved Sandwich Panels With Honeycomb Cores," Compos. Struct., 17(2), pp. 95-111.

[29] Choi, J. B., and Lakes, R. S., 1991, "Design of a Fastener Based on Negative Poisson's Ratio Foam," Cell. Polym., 10(3), pp. 205-212.

[30] Martin, J., Heyder-Bruckner, J.-J., Remillat, C., Scarpa, F., Potter, K., and Ruzzene, M., 2008, "The Hexachiral Prismatic Wingbox Concept," Physica Status Solidi (b), 245(3), pp. 570-577.

[31] Bertoldi, K., Reis, P., Willshaw, S., and Mullin, T., 2010, "Negative Poisson's Ratio Behavior Induced by An Elastic Instability," Adv. Mater., 22(3), pp. 361-366.

[32] Agnese, F., Remillat, C., Scarpa, F., and Payne, C., 2015, "Composite Chira Shear Vibration Damper," Compos. Struct., 132, pp. 215-225.

[33] Jang, K.-I., Chung, H. U., Xu, S., Lee, C. H., Luan, H., Jeong, J., Cheng, H., Kim, G.-T., Han, S. Y., Lee, J. W., Kim, J., Cho, M., Miao, F., Yang, Y., Jung, H. N., Flavin, M., Liu, H., Kong, G. W., Yu, K. J., Rhee, S. I., Chung, J., Kim, B., Kwak, J. W., Yun, M. H., Kim, J. Y., Song, Y. M., Paik, U., Zhang, Y., Huang, Y., and Rogers, J. A., 2015, "Soft Network Composite Materials With Deterministic and Bio-inspired Designs," Nat. Commun., 6, p. 6566.

[34] Lipsett, A. W., and Beltzer, A. I., 1988, "Reexamination of Dynamic Problems of Elasticity for Negative Poisson's Ratio," J. Acoust. Soc. Am., 84(6), pp. 2179 2186

[35] Howell, B., Prendergast, P., and Hansen, L., 1994, "Examination of Acoustic Behavior of Negative Poisson's Ratio Materials," Appl. Acoust., 43(2), pp. 141-148.

[36] Chen, C. P., and Lakes, R. S., 1996, "Micromechanical Analysis of Dynamic Behavior of Conventional and Negative Poisson's Ratio Foams," ASME J. Eng. Mater. Technol., 118(3), pp. 285-288.
[37] Scarpa, F., Ciffo, L. G., and Yates, J. R., 2004, "Dynamic Properties of High Structural Integrity Auxetic Open Cell Foam," Smart Mater. Struct., 13(1), p. 49. 38] Chekkal, I., Bianchi, M., Remillat, C., Becot, F.-X., Jaouen, L., and Scarpa, F., 2010, "Vibro-Acoustic Properties of Auxetic Open Cell Foam: Model and Experimental Results," Acta Acustica United Acustica, 96(2), pp. 266-274.

[39] Spadoni, A., Ruzzene, M., Gonella, S., and Scarpa, F., 2009, "Phononic Properties of Hexagonal Chiral Lattices," Wave Motion, 46(7), pp. 435-450.

[40] Auffray, N., Dirrenberger, J., and Rosi, G., 2015 "A Complete Description of Bi-dimensional Anisotropic Strain-Gradient Elasticity," Int. J. Solids. Struct., 69-70, pp. 195-210.

[41] Rosi, G., and Auffray, N., 2016, "Anisotropic and Dispersive Wave Propagation Within Strain-Gradient Framework," Wave Motion, 63, pp. 120-134.

[42] Poncelet, M., Somera, A., Morel, C., Jailin, C., and Auffray, N., 2018, "An Experimental Evidence of the Failure of Cauchy Elasticity for the Overall Modeling of a Non-Centro-Symmetric Lattice Under Static Loading," Int. J. Solids Struct., 147, pp. 223-237.

[43] Scarpa, F., Yates, J. R., Ciffo, L. G., and Patsias, S., 2002, "Dynamic Crushing of Auxetic Open-Cell Polyurethane Foam," Proc. Inst. Mech. Eng. Part C J. Mech. Eng. Sci., 216(12), pp. 1153-1156.

[44] Liu, W., Wang, N., Luo, T., and Lin, Z., 2016, "In-Plane Dynamic Crushing of Re-Entrant Auxetic Cellular Structure," Mater. Des., 100, pp. 84-91.

[45] Li, T., Chen, Y., Hu, X., Li, Y., and Wang, L., 2018, "Exploiting Negative Poisson's Ratio to Design 3d-Printed Composites With Enhanced Mechanical Properties," Mater. Des., 142, pp. 247-258.

[46] Novak, N., Starčevič, L., Vesenjak, M., and Ren, Z., 2019, "Blast Response Study of the Sandwich Composite Panels With 3d Chiral Auxetic Core," Composite Structures, 210, pp. 167-178.

[47] Chan, N., and Evans, K. E., 1998, "Indentation Resilience of Conventional and Auxetic Foams," J. Cell. Plast., 34(3), pp. 231-260.

[48] Gilat, R., and Aboudi, J., 2013, "Behavior of Elastoplastic Auxetic Microstructural Arrays," Materials, 6(3), pp. 726-737.

[49] Ghaedizadeh, A., Shen, J., Ren, X., and Xie, Y. M., 2016, "Tuning the Performance of Metallic Auxetic Metamaterials by Using Buckling and Plasticity," Materials, 9(54), pp. 1-17.

[50] Bornert, M., Bretheau, T., and Gilormini, P., 2001, Homogénéisation En Mécanique Des Matériaux, Tome 1 : Matériaux Aléatoires élastiques Et Milieux Périodiques, Hermès, Paris.

[51] Besson, J., Cailletaud, G., Chaboche, J.-L., Forest, S., and Blétry, M., 2010, Non-Linear Mechanics of Materials, Vol. 167 of Solid Mechanics and Its Applications, Springer, New York

[52] Jiang, M., Jasiuk, I., and Ostoja-Starzewski, M., 2002, “Apparent Elastic and Elastoplastic Behavior of Periodic Composites," Int. J. Solids Struct., 39(1), pp. 199-212

[53] Cailletaud, G., Forest, S., Jeulin, D., Feyel, F., Galliet, I., Mounoury, V., and Quilici, S., 2003, "Some Elements of Microstructural Mechanics," Comput. Mater. Sci., 27(3), pp. 351-374.

[54] Yuan, Z., and Fish, J., 2008, "Toward Realization of Computational Homogenization in Practice," Int. J. Numer. Methods Eng., 73(3), pp. 361-380.

[55] Fritzen, F., Forest, S., Kondo, D., and Böhlke, T., 2013, "Computational Homogenization of Porous Materials of Green Type," Computat. Mech., 52(1), pp. 121-134

[56] Geers, M. G. D., and Yvonnet, J., 2016, "Multiscale Modeling of Microstructure-Property Relations," MRS Bull., 41(8), pp. 610-616.

[57] Evans, A. G., Hutchinson, J. W., Fleck, N. A., Ashby, M. F., and Wadley, H. N. G., 2001, "The Topological Design of Multifunctional Cellular Metals," Prog. Mater. Sci., 46(3-4), pp. 309-327.

[58] Deshpande, V. S., Ashby, M. F., and Fleck, N. A., 2001, "Foam Topology: Bending Versus Stretching Dominated Architectures," Acta Mater., 49(6), pp. $1035-1040$

[59] Deshpande, V. S., Fleck, N. A., and Ashby, M. F., 2001, "Effective Properties of the Octet-Truss Lattice Material," J. Mech. Phys. Solids, 49(8), pp. 1747-1769.

[60] Kooistra, G. W., Deshpande, V. S., and Wadley, H. N., 2004, "Compressive Behavior of Age Hardenable Tetrahedral Lattice Truss Structures Made From Aluminium," Acta Mater., 52(14), pp. 4229-4237.

[61] Ashby, M. F., 2006, "The Properties of Foams and Lattices," Philos. Trans. R. Soc. A Math., Phys. Eng. Sci., 364(1838), pp. 15-30.

[62] Fleck, N. A., Deshpande, V. S., and Ashby, M. F., 2010, "Micro-architectured Materials: Past, Present and Future," Proc. R. Soc. A Math., Phys. Eng. Sci., 466(2121), pp. 2495-2516.

[63] Vigliotti, A., and Pasini, D., 2012, "Stiffness and Strength of Tridimensional Periodic Lattices," Comput. Methods Appl. Mech. Eng., 229, pp. 27-43.

[64] Vigliotti, A., and Pasini, D., 2013, "Mechanical Properties of Hierarchical Lattices," Mech. Mater., 62, pp. 32-43.

[65] Zok, F. W., Latture, R. M., and Begley, M. R., 2016, "Periodic Truss Structures," J. Mech. Phys. Solids, 96, pp. 184-203.

[66] Latture, R. M., Begley, M. R., and Zok, F. W., 2018, "Design and Mechanical Properties of Elastically Isotropic Trusses," J. Mater. Res., 33(3), pp. 249-263.

[67] Latture, R. M., Rodriguez, R. X., Holmes, L. R., Jr., and Zok, F. W., 2018, "Effects of Nodal Fillets and External Boundaries on Compressive Response of an Octet Truss," Acta Mater., 149, pp. 78-87.

[68] Tancogne-Dejean, T., and Mohr, D., 2018, "Elastically-Isotropic Truss Lattice Materials of Reduced Plastic Anisotropy," Int. J. Solids Struct., 138, pp. 24-39.

[69] Bonatti, C. and Mohr, D. 2019, "Mechanical Performance of Additively-Manufactured Anisotropic and Isotropic Smooth Shell-Lattice Materials: Simulations \& Experiments," J. Mech. Phys. Solids, 122, pp. 1-26.

[70] Maxwell, J. C., 1864, "On the Calculation of the Equilibrium and Stiffness of Frames,” London, Edinburgh, Dublin Philos. Mag. J. Sci., 27(182), pp. 294-299. 
[71] Calladine, C. R., 1978, "Buckminster Fuller's 'Tensegrity' Structures and Clerk Maxwell's Rules for the Construction of Stiff Frames," Int. J. Solids Struct. 14(2), pp. 161-172.

[72] Pellegrino, S., and Calladine, C. R., 1986, "Matrix Analysis of Statically and Kinematically Indeterminate Frameworks," Int. J. Solids Struct., 22(4), pp. $409-428$.

[73] Mitschke, H., Schury, F., Mecke, K., Wein, F., Stingl, M., and Schröder-Turk, G., 2016, "Geometry: The Leading Parameter for the Poissons Ratio of Bending-Dominated Cellular Solids," Int. J. Solids Struct., 100-101, pp. 1-10.

[74] Rayneau-Kirkhope, D., 2018, "Stiff Auxetics: Hierarchy as a Route to Stiff, Strong Lattice Based Auxetic Meta-materials," Sci. Rep., 8, p. 12437.

[75] Weaver, P. M., and Ashby, M. F., 1996, "The Optimal Selection of Material and Section-Shape," J. Eng. Design, 7(2), pp. 129-150.

[76] Gibson, L. J., and Ashby, M. F., 1999, Cellular Materials, Cambridge University Press, Cambridge.

[77] Ashby, M., 2013, "Designing Architectured Materials," Scr. Mater., 68(1), pp. 4-7.

[78] Warmuth, F., Wormser, M., and Körner, C., 2017, "Single Phase 3d Phononic Band Gap Material," Sci. Rep., 7(1), p. 3843.

[79] Bertoldi, K., Reis, P. M., Willshaw, S., and Mullin, T., 2010, “Negative Poisson's Ratio Behavior Induced by an Elastic Instability," Adv. Mater., 22(3), pp. 361366.

[80] Babaee, S., Shim, J., Weaver, J., Chen, E., Patel, N., and Bertoldi, K., 2013, “3D Soft Metamaterials With Negative Poisson's Ratio," Adv. Mater., 25(36), pp. 5044-5049.

[81] Ren, X., Shen, J., Ghaedizadeh, A., Tian, H., and Xie, Y. M., 2015, "Experiments and Parametric Studies on 3d Metallic Auxetic Metamaterials With Tuneable Mechanical Properties," Smart Materials Struct., 24(9), p. 095016.

[82] Ren, X., Shen, J., Tran, P., Ngo, T. D., and Xie, Y. M., 2018, "Design and Characterisation of a Tuneable 3d Buckling-Induced Auxetic Metamaterial," Mater. Des., 139, pp. 336-342.
[83] Bacigalupo, A., and Gambarotta, L., 2014, "Homogenization of Periodic Hexaand Tetrachiral Cellular Solids," Compos. Struct., 116(1), pp. 461-476.

[84] Bacigalupo, A., and De Bellis, M. L., 2015, "Auxetic Anti-tetrachiral Materials: Equivalent Elastic Properties and Frequency Band-Gaps," Compos. Struct., 131, pp. 530-544.

[85] Mukhopadhyay, T., and Adhikari, S., 2016, "Effective In-Plane Elastic Properties of Auxetic Honeycombs With Spatial Irregularity," Mech. Mater., 95, pp. 204-222.

[86] Geuzaine, C., and Remacle, J.-F., 2009, "Gmsh: A 3-D Finite Element Mesh Generator With Built-In Pre- and Post-Processing Facilities," Int. J. Numer. Meth. Eng., 79(11), pp. 1309-1331.

[87] Sab, K., 1992, "On the Homogenization and the Simulation of Random Materials," Eur. J. Mech., A/Solids, 11(5), pp. 585-607.

[88] Kanit, T., Forest, S., Galliet, I., Mounoury, V., and Jeulin, D., 2003, "Determination of the Size of the Representative Volume Element for Random Composites: Statistical and Numerical Approach," Int. J. Solids Struct., 40(13-14), pp. 3647-3679.

[89] Bunge, H.-J., 1982, Texture Analysis in Materials Science: Mathematical Methods, Elsevier, New York,

[90] Pouzet, S., Peyre, P., Gorny, C., Castelnau, O., Baudin, T., Brisset, F., Colin, C., and Gadaud, P., 2016, "Additive Layer Manufacturing of Titanium Matrix Composites Using the Direct Metal Deposition Laser Process," Mater. Sci. Eng. A., 677, pp. 171-181.

[91] Warmuth, F., Osmanlic, F., Adler, L., Lodes, M. A., and Körner, C., 2016 , "Fabrication and Characterisation of a Fully Auxetic 3d Lattice Structure Via Selective Electron Beam Melting," Smart Mater. Struct., 26(2), p. 025013.

[92] Auffray, N., 2014, "Analytical Expressions for Odd-Order Anisotropic Tensor Dimension," Comptes Rendus Mécaniques, 342(5), pp. 284-291.

[93] Auffray, N., He, Q. C., and Le Quang, H., 2019, "Complete Symmetry Classification and Compact Matrix Representations for 3D Strain Gradient Elasticity,” Int. J. Solids Struct., 159, pp. 197-210. 